\title{
FluidScape: Research in Parametric Concrete Formwork
}

\author{
Nathan Howe \\ Kansas State University, USA \\ nhowe@ksu.edu
}

\begin{abstract}
Given the piecemeal nature of concrete formwork, the phenomenon of the monolithic result relies on elegant detailing, material choice, and formal continuity. Creating complex form out of concrete poses an intriguing problem, where these disparate constructive elements must be dynamic and flexible. This paper describes the design process from conception to prototyping for a public spray park competition project. The challenges inherent in the proposed forms included subtle reveals and ledges for water to flow down. Achieving the desired finish for the concrete requires a precise, digitally-generated form, particular materials and fabrication sequence. The project design and fabrication gives parametric control to Computer Numerically Controlled (CNC) fabrication tools for an output of fluid form.
\end{abstract}

Keywords: Parametric; Digital fabrication; Scripting; Concrete; Formwork.

\section{Introduction}

FluidScape was first conceived as a competition entry for a small neighborhood park in Philadelphia. It has since won and been commissioned for construction documentation. Prior to completing the documentation, a series of explorations in fabrication techniques was required to balance the integrity of the design with the economics of creating formwork, while controlling the final finish of the concrete and creating a clear installation process. This paper focuses on the serpentine concrete wall form at the heart of the design. It creates the basis for the research of how the fabrication process and the fluidity of the parametric controls of the form came into alignment to facilitate testing. Various means of fabrication will be explained, including failed attempts and successful methods. It will also describe the nature of the formwork materiality, its strength and flexibility, and how this information was integral to the parameters of the design. This is an ongoing research project, and as professional fabricators are brought on board the project will continue to evolve. This paper will conclude with a forecast of other concrete construction methods being explored, and how these other approaches may change the parametric methodology, while holding true to the main objectives of the design.

Parametric design has become an ever more important field in architecture. Use of this advanced technique has led to a much more controlled notion of design and has established parameters for performance and construction methodology (Rahim, 2006). FluidScape continues within this paradigm shift. Its parametric model allowed there to be a creative cycle where knowledge from prototypes immediately affected change within the more complex relationship of part to whole, and provided a feedback loop.

To bring the project in line with construction logic, every form was designed using ruled surfaces. This is much like Antonio Gaudi's geometric logic in many of his projects, most famously in the Segrada Familia Cathedral. Gaudi used hyperbolic paraboloid forms in this design, which are simply a specific type of ruled surface based on twisted form (Burry, 2011). In FluidScape, these ruled surfaces were tested not only for their aesthetic integrity, but also to determine whether they were developable. Developable surfaces can be unrolled flat for Computer Numerically Controlled (CNC) cutting. This created an inherent logic of constructability which grounded the design.

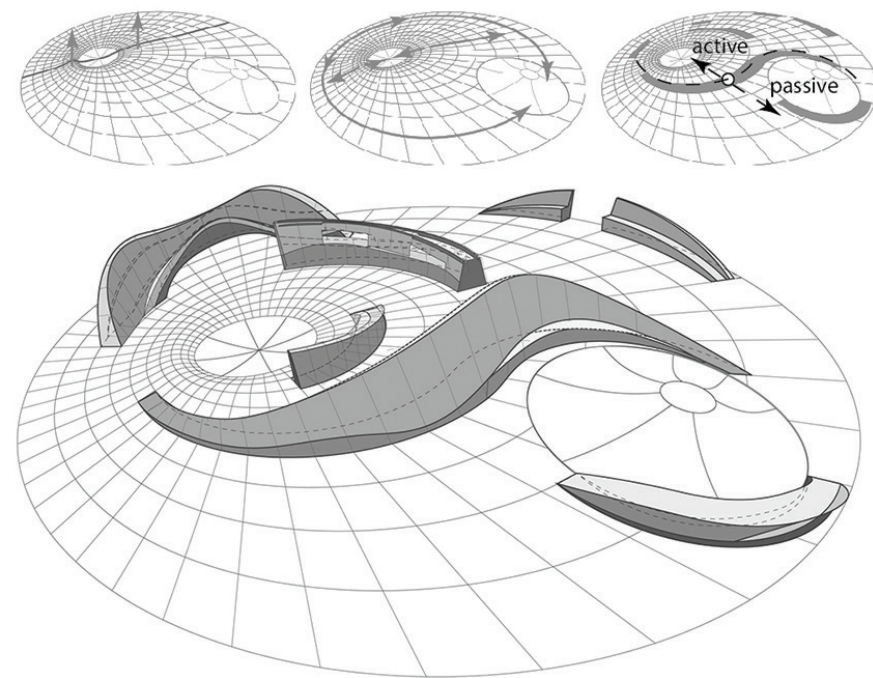

Figure 1: This aerial perspective view lays out the basic geometric pattern of the design as well as the design evolution as the base surface is manipulated, the water flow is determined and the various forms are composed in an interlocking spatial pattern. 


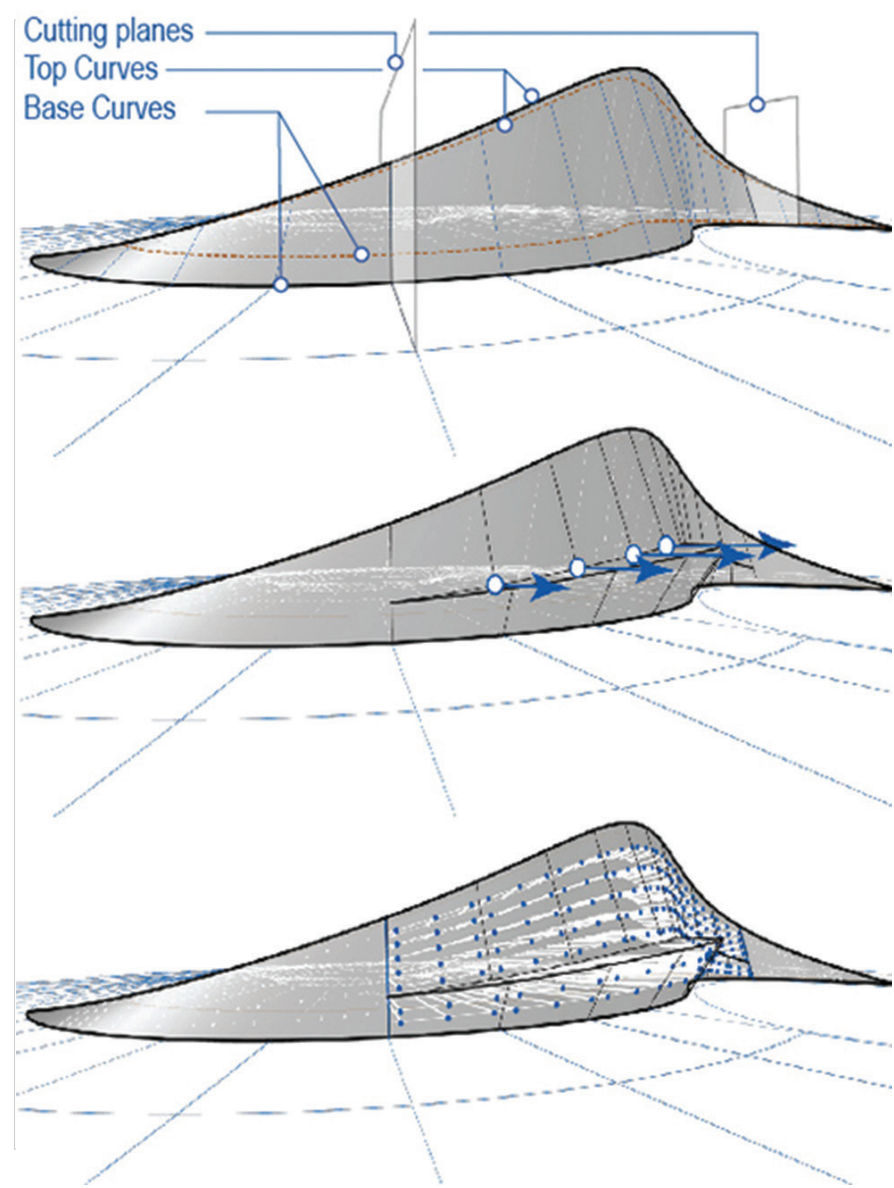

Figure 2: The top image denotes the placement of cutting planes which divide it into manageable pieces. The middle piece can then form deviations for seating seen in the middle image. The bottom image shows how the reinforcing pattern for formwork is incorporated into the design logic.

\section{Parametric Logic}

The intrinsic beauty of the parametric model is that fabrication can be developed alongside form. In Archi-Union Architects (2012) Tea House, the process of designing was also forged with a parametric model. In the Tea House, complex geometry had to be formulated into a constructible method with only rather unsophisticated construction skills available. In this project, ruled surfaces were established in the computer and then reestablished on site through a series of string lines. This created a matrix that was then board-formed with dimensional lumber.

The forms seen in FluidScape are made up of ruled surfaces that tie into the base geometry (Figure 1). Radial lines define the logic for form placement, as well as beginning and end points, and hold the entire design within the bounds of explicit logic. The largest of the spray park forms generated by these rules, the serpentine wall, reaches $40 \mathrm{ft}$. long $\times 4 \mathrm{ft}$. tall $\times 5 \mathrm{ft}$. wide. The sheer size and precision required by the forms within the larger design insist on the clear control of a parametrically-driven construction strategy.
To create the serpentine wall of FluidScape, perhaps the most straightforward method to produce formwork would be milling solid foam on a 5-axis CNC. However, the cost of machining and cost of the large amount of form material made this infeasible. By employing ruled surface geometry, FluidScape's premise of construction became creating formwork from flat stock sheet metal shaped back into molds.

The basic logic of the serpentine wall's design evolution is seen in Figure 2. The overall form begins with four primary curves, two on the base and two at the top. With just these four curves, the logic defines smaller parts where the radial lines slice through the form, and create "isocurves" across the surface. Isocurves are the delineation of the surface cutting along the surface oriented in the $U$ and $V$ directions of the surface. These isocurves serve as the ordering logic of deviations, sectioning and tie rod patterns.

Figure 2 also depicts how the form can be broken into three parts using the isocurves. This is necessary because the form is nearly 40 $\mathrm{ft}$. long and the parts would be too large for most $2 \frac{1}{2}$-axis CNC fabricators, and the pieces would reach a size unwieldy for fabrication into formwork. This separation into parts also provided a design opportunity to explore deviations. For example, within the middle section, the surface peels away in the lower half and creates a bench that sweeps from a child height for sitting to an adult size. These deviations become important to add more function and delight to the overall design, and are all controlled through the parametric model.

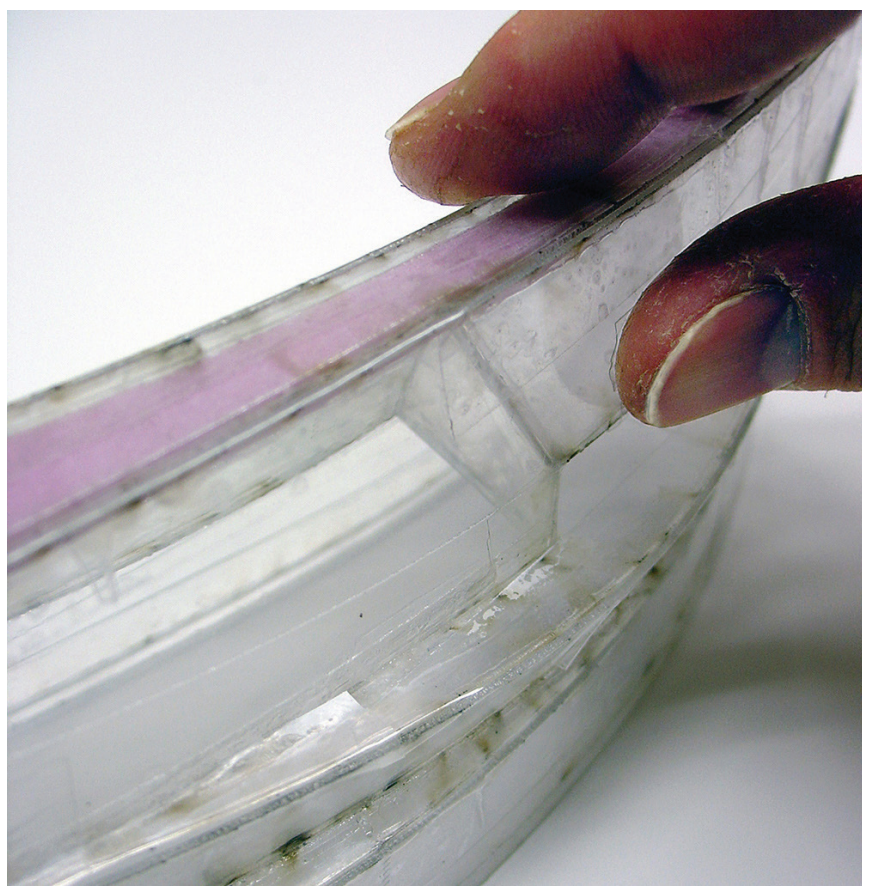

Figure 3: Fabricated using a CNC laser cutter, this $3 / 4$ " scale acrylic model represents a complete formwork for a concrete pour. 


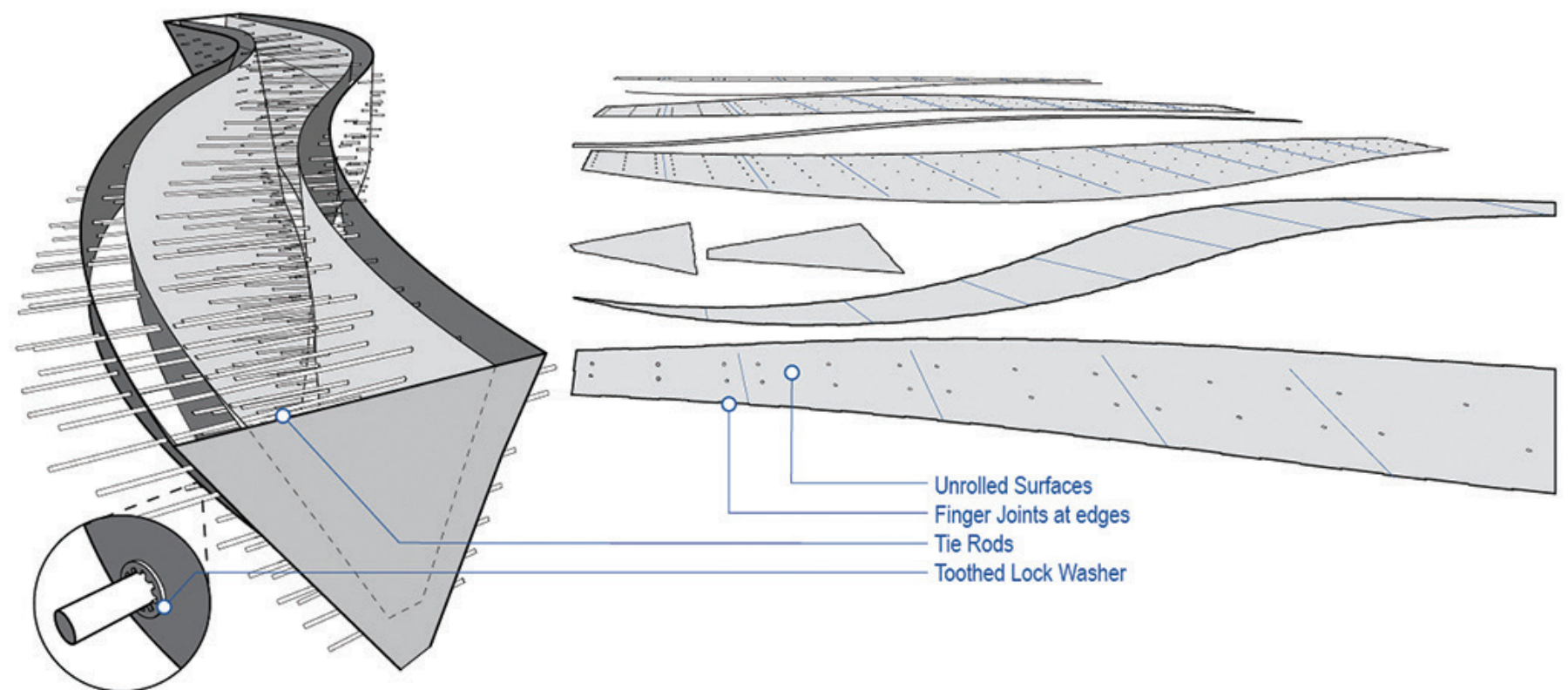

Figure 4: This inverted mold was exploring the accuracy of the finger joint method. This joint eased difficulty of fabricating the forms. Within the inverted form work, the tie rods pierced the entire form and then toothed lock washers held the surfaces in place.

To test the basic logic of the ruled surface, a series of small scale models were created. This helped to better constrain the design's surfaces so they did not get too extreme in their curvature to the point of being undevelopable (see Figure 3). These models serve as proof of concept and are analogous to the first strategy of construction method where the unrolled surfaces could be stitched back together to create the formwork for the concrete pour. In this case acrylic was used as the formwork material. These forms were then inverted for concrete pours.

\section{Inverted Mold Concept}

In FluidScape, the first fabrication technique tested was an inverted mold. Unlike traditional formwork where its rigidity is found mostly in bracing and formwork strength, this type of complex geometry creates a rigid system independent of bracing. Using the principle that Salvadori (1980) discusses in curved surface as form-resistant structures, FluidScape's forms use curved surfaces to maintain rigidity in the concrete pour process. This, however, will not keep the form from buckling and deforming completely, especially given the thin stock being used in formwork.

In this concept, tie rods were used to perform the critical role of holding the form in perfect registry with the computer model design. The pattern of the tie rods can be seen in the bottom portion of Figure 2. This pattern was initially created based on an intuitive understanding of what is necessary to hold the sheet metal rigid, and tie rods were spaced every 6 to 9 inches. The pattern also considered the tracery of geometry of the isocurves and sectioning of the large forms. The pattern established itself as inset from the isocurves, and allowed the eventual seams caused by the sectioning to not interrupt the flow patterns the rods gave the overall serpentine form.
Once the tie rod pattern was established, the surfaces were unrolled and readied for CNC cutting. To help with surface registry, a script was created to design a finger joint method of stitching the surfaces together. This finger joint allowed for a more direct fabrication of formwork process than our first few prototypes allowed. This finger joint script compared all edges and paired according to equal lengths. Once the pairings were established, the finger joints were controlled by length and depth parameters. An example of an early prototype of the finger joint can be seen in Figure 4 on the unrolled surfaces and in Figure 5 fully assembled.

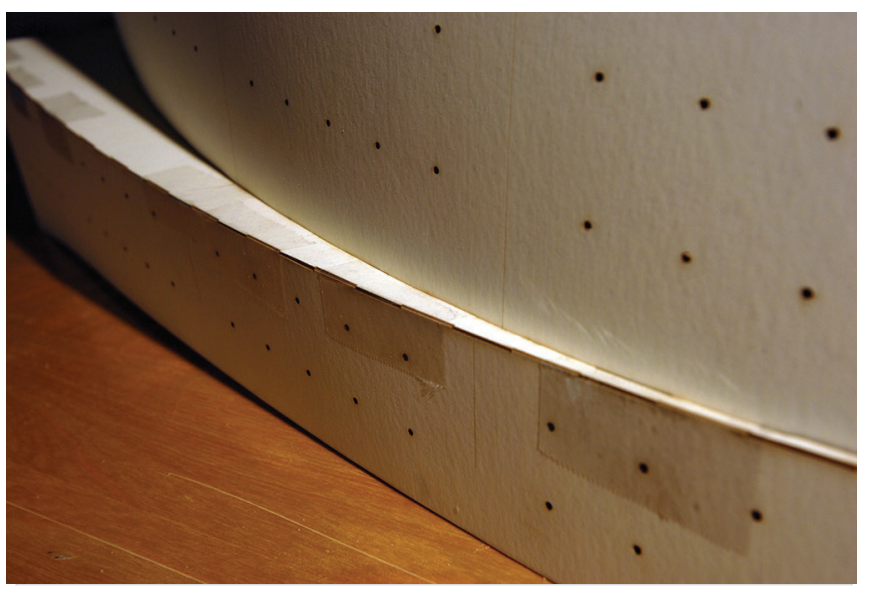

Figure 5: This prototype was exploring the accuracy of the finger joint method. This joint eased difficulty of fabricating the forms.

With a clear method established for constructing the concrete formwork, the tie rod and finger joint thesis was tested. In initial tests, a small-bed laser cutter was used to cut acrylic sheet material at a scale of $4 \mathrm{in}$. = $1 \mathrm{ft}$. The middle section of the serpentine wall was inverted as seen in Figure 4 (due to the size of laser bed, the form had to be further sectioned in half, as seen in Figure 6). For this test, $0.8 \mathrm{~mm}$. acrylic sheet material was used. At 
this scale this material has a similar flexibility to $16-18$ gauge sheet metal would have at full scale. Acrylic rods were employed for the tie rods, but toothed lock washers could not be used at this scale, so each rod was glued. The thickness of the concrete was conservatively made 4 " thick to scale, and thicker in some places, as seen in Figure 6. At full scale, glass fiber reinforced concrete (GFRC) would be used. This should allow for the amount of concrete to be minimized; in most cases as thin as 1 " thick. This would provide reinforcing with navigating steel rebar and result in less hydrostatic pressure on the formwork.

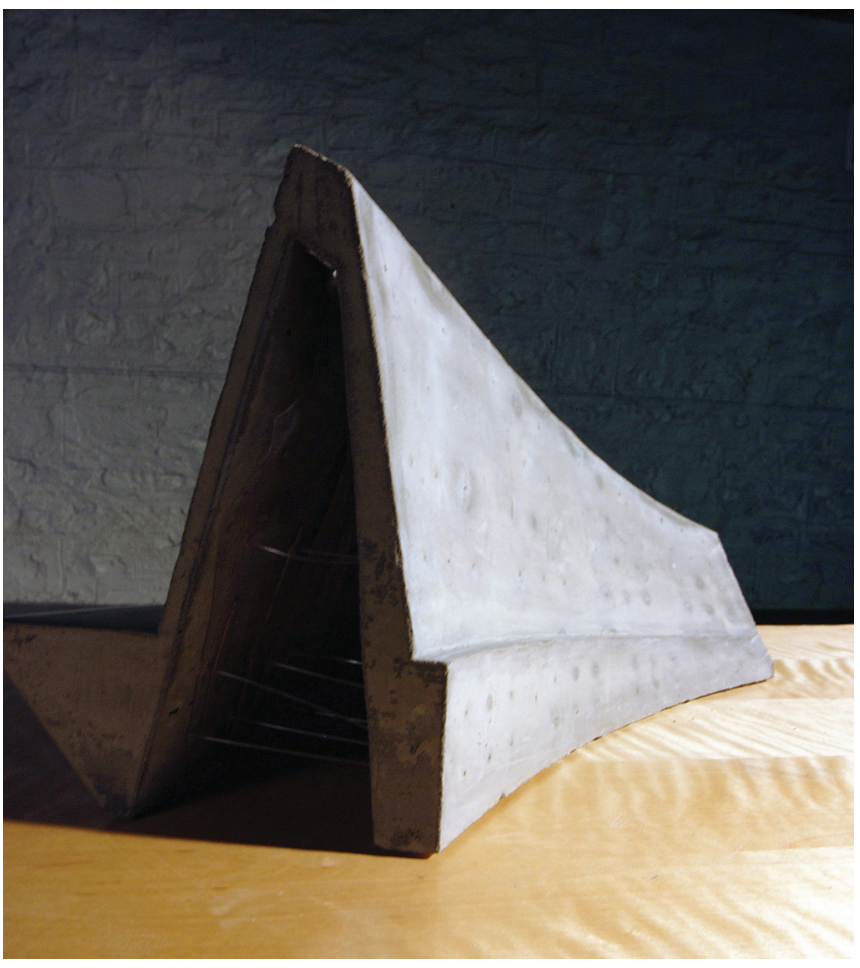

Figure 6: This model tests the tie rod pattern. Acrylic rods hold the acrylic sheet in equilibrium during the concrete pour.

The result of this prototype was quite remarkable given the relative flexibility of the acrylic and the thickness of the concrete walls. Under the hydrostatic pressure placed on the formwork model, the rods performed perfectly. It was noted that the interior sides of the form tended to deform, as the rods in the cavity were in compression. However, this deviation only affected the interior cavity, and was irrelevant to exterior finish. Figure 6 demonstrates proof of concept. A slight deviation near the isocurves traceries occurred because the rods spaced on either side did not come close enough to avoid slight rippling of the surface. Using the parametric model, an immediate fix was made, and output was available within moments of the change to bring the reinforcing pattern closer to the isocurves.

\section{Present Explorations}

At present, some of this has become an academic exercise. With only a full-scale mock-up left before the concept is proven, the project is in a hold pattern. The inverted mold method will work, but finding a concrete fabricator willing to join the experimentation has been difficult. With an improved understanding of what the concrete industry is willing to engage, and in order to make the project feasible for our client, there has been a flurry of other construction methods explored. Each of these required a degree of parametric processing to bring the form and fabrication logic into alignment.

\section{Clam shell concept}

In Figure 7, a variation on the clam shell method, common in mold-making, can be seen. With the goal to make the form walls as thin as possible, this concept best engages a spray-on GFRC methodology. The forms are spit in half and separated with a fabricated sheet metal mold. The process of mold making is created in much the same manner as described previously with the inverted mold. When GFRC is applied as a spray, a thin layer becomes as strong as a much thicker reinforced concrete system. Spraying GFRC into the molds would allow a $3 / 4$ " thickness to be used in this clam shell technique. Much less structure for the formwork is needed, and tie rods are no longer necessary.

The clam mold has a clear advantage over the inverted mold in finish quality, because absolutely no bubbles can form due to the spray-on process. In the inverted mold method, the concrete must be vibrated to get bubbles to float to the top. Testing found that bubbles got caught within deviations such as the benches, and this imperfection was exposed on the final finish.

\section{Shotcrete concept}

The shotcrete concept is seen as the most financially feasible solution. If adopted, steel framework will be fabricated to provide a backing for concrete and fin sections of stainless steel at the isocurve locations. This gives a continual registration for shotcrete to be applied. Figure 7 illustrates how even the rebar pattern has been cut into the fins for ease of fabrication.

The major issue for this construction method is the unpredictability. The design idea for this project was to create a fabrication strategy where the final finish of the concrete could be pristine. This concept leaves final finish up to the contractor. The fins provide a guide for the skim coat, but to transfer a perfect curved surface between the fins would require a craftsman, not just a contractor. This is not ideal, as quality control is taken out of the designer's hands.

Another issue will be the complication of form. A few of the form types have a formal complexity which may have to be simplified to allow for the shotcrete and troweling process to have success. 

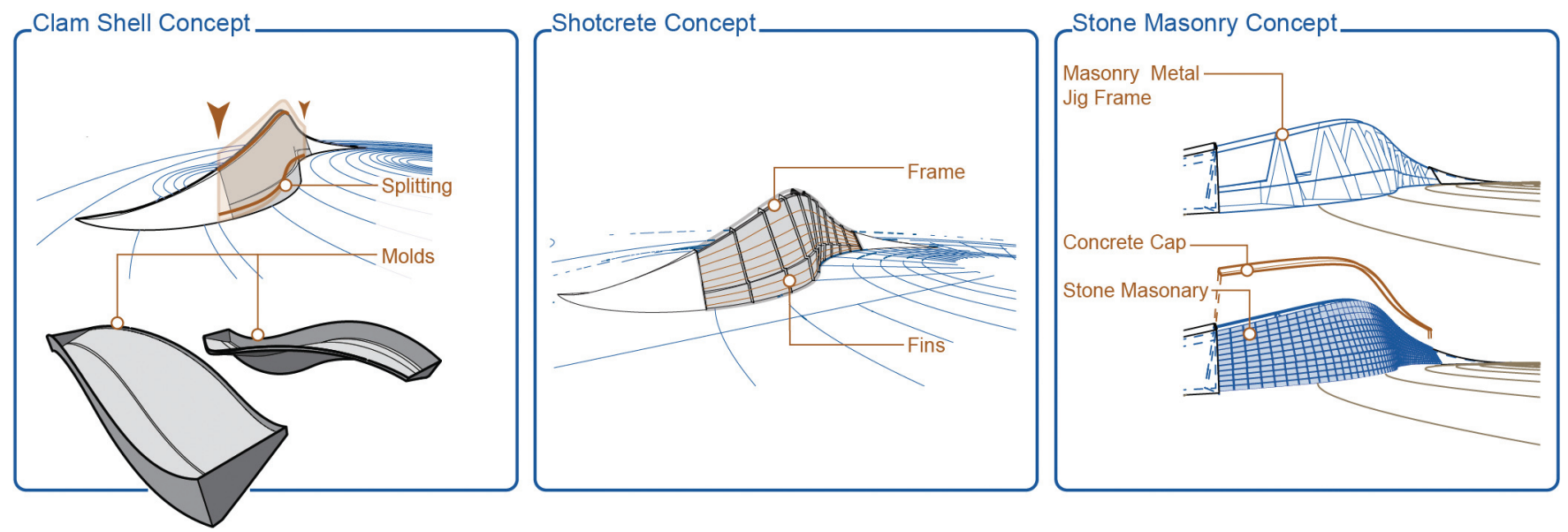

Figure 7: These three concepts represent other methods currently being explored to find a balance between design quality and economic feasibility for the client.

\section{Stone masonry concept}

While searching for concrete fabricators willing to tackle the project, it was realized that Philadelphia has excellent masons. It is possible this craft could create a very different look for the project, but absolutely fall in line with the vision. This concept also makes use of a steel framework for anchoring the masonry. The fins would also give registration for the forms the masons would need to follow. While the final artifact is left up to the craftsman, the strong history of this craft in Philadelphia makes this option more palatable. As noted in the shotcrete concept, a potential limiting factor is the degree of complexity which could be registered and built with masonry. This may necessitate a simplifying of form.

\section{Conclusion}

The research and testing of FluidScape has continued to establish a fusion of the design process and the act of fabrication. Holding the connective logic of the model allows the designer to act as the puppet master, or perhaps more appropriately, the master builder. However, as the final mark is to actually build the design, there has to be a clear connection with the final fabricator. This is a balancing act between experimentation and what the industry can and is willing to construct.

\section{References}

Archi-Union Architects. (2012). Tea House [No. 1436 Jungong Road, Yangpu District, Shanghai] - architect: Archi-Union Architects. Space, pp. 42-47.

Burry, M. (2011, July/August). Geometry Working Beyond Effect. Architectural Design, 80-89.

Rahim, A. (2006). Catalytic Formations: Architecture and Digital Design. New York Taylor \& Francis.

Salvadori, M. (1980). Why Buildings Stand Up. New York: Norton. 